\title{
Quality of life in patients with coronary artery disease treated with coronary artery bypass grafting and hybrid coronary revascularization
}

\author{
Karolina Gierszewska ${ }^{1}$, Izabela Jaworska ${ }^{2}$, Michał Skrzypek ${ }^{3}$, \\ Mariusz Gąsior ${ }^{1}$, Robert Pudlo ${ }^{4}$
}

${ }^{1} 3^{\text {rd }}$ Department of Cardiology, SMDZ in Zabrze, Medical University of Silesia, Katowice, Silesian Centre for Heart Disease in Zabrze, Poland

${ }^{2}$ Department of Cardiac, Vascular and Endovascular Surgery and Transplantology, SMDZ in Zabrze, Medical University of Silesia in Katowice, Silesian Centre for Heart Disease in Zabrze, Poland ${ }^{3}$ Medical University of Silesia, School of Public Health in Bytom, Department of Biostatistics, Poland ${ }^{4}$ Department of Psychiatry in Tarnowskie Gory, School of Medicine with the Division of Dentistry in Zabrze, Medical University of Silesia in Katowice, Poland

\begin{abstract}
Background: Patients with stable coronary artery disease (CAD) have a worse quality of life (QoL) in comparison to patients without stable CAD. Standardized questionnaires are used in evaluation of QoL. Hybrid coronary revascularization (HCR) is a recently-introduced, minimally invasive option for patients requiring revascularization for coronary lesions. The aim of this study was to assess healthrelated quality of life (HRQoL) in patients with multivessel CAD (MVCAD), according to the mode of revascularization: coronary artery bypass grafting (CABG) or HCR, using the generic SF-36 v.2 questionnaire.

Methods: From November 2009 to July 2012, 200 patients from POLMIDES study with diagnosed $M V C A D$ and were referred for conventional $C A B G$ were randomized to $H C R(n=98)$ or $C A B G$ ( $n=102)$ groups in 1:1 ratio. HRQoL were measured at two time points: hospital admission and 12-month follow up. The primary endpoint was the difference in HRQoL after the procedure.

Results: Both groups showed the same improvement of HRQoL: in HCR group: 13.5 (3.82-22.34) vs. $C A B G$ group: 10.48 (2.46-31.07); $p=0.76$.

Conclusions: $H R Q o L$ in patients after both modes of revascularization significantly improved after 12 months in all domains. (Cardiol J 2018; 25, 5: 621-627)

Key words: quality of life, coronary artery disease
\end{abstract}

\section{Introduction}

Stable coronary artery disease (CAD) has a negative impact on physical and psychological functioning of patients. It has been proven that patients with stable CAD have a worse quality of life (QoL) in comparison to patients without stable
CAD [1]. The holistic approach to patient health has lead to a dynamic growth of interest in research concerning QoL in cardiovascular diseases. From the 90 s of the twentieth century health-relalet quality of life (HRQoL) has become an important health outcome indicator [2-4]. Standardized questionnaires are used in the evaluation of QoL.

Address for correspondence: Karolina Gierszewska, MD, $3^{\text {rd }}$ Department of Cardiology, SMDZ in Zabrze, Medical University of Silesia, Katowice, Silesian Centre for Heart Disease in Zabrze, ul. M. Curie-Skłodowskiej 9, 41-800 Zabrze, Poland, tel: +48 3237338 60, fax: +48 3237338 19, e-mail: gierlaszynska@gmail.com; sek.med@sccs.pl 


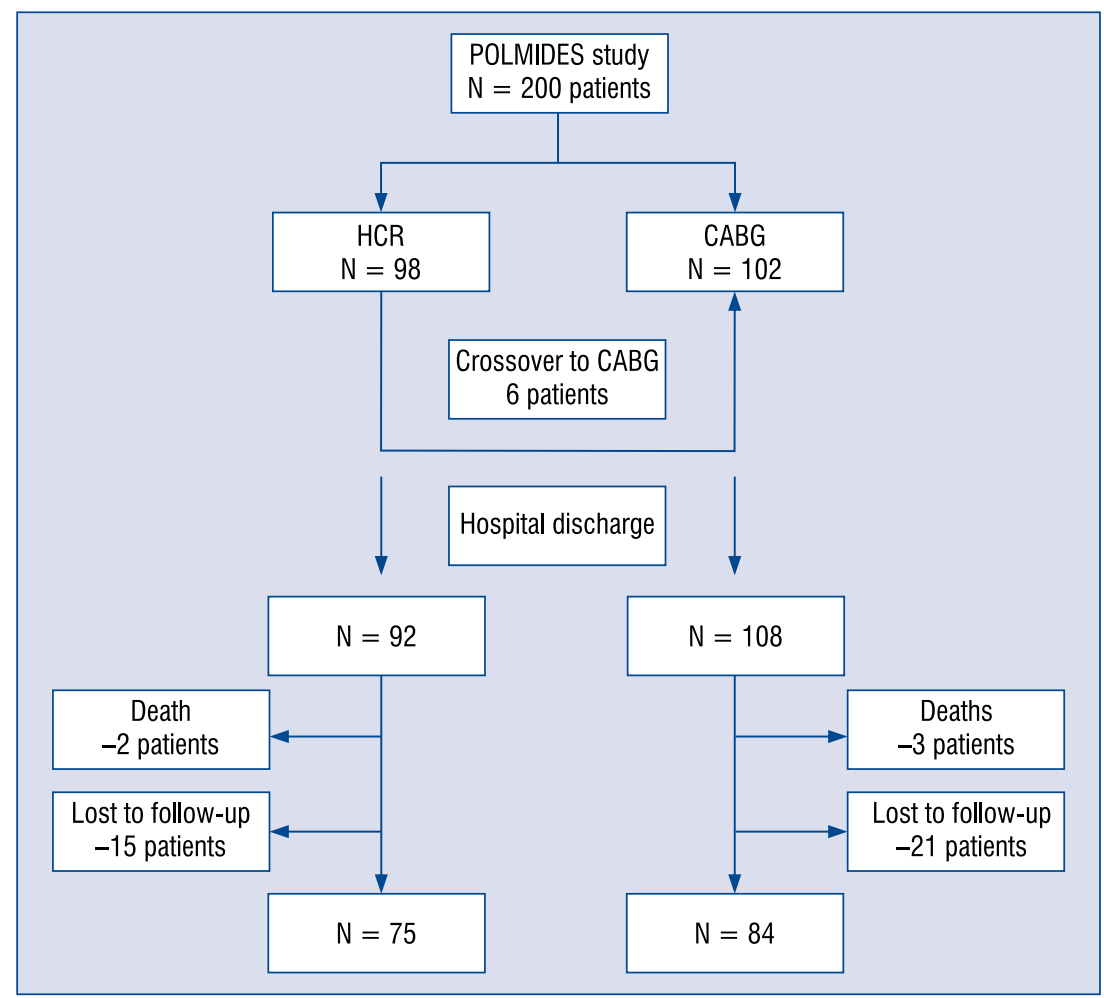

Figure 1. Study flow chart; CABG — coronary artery bypass grafting; HCR — hybrid coronary revascularization.

There are two types of questionnaires: generic and disease-specific. One of the most widely used generic health status instruments to assess HRQoL is the Medical Outcomes Study Short Form (SF-36) questionnaire and it has been studied in many populations, also in patients with CAD and valvular heart disease [5-9]. Many studies have shown that coronary artery bypass grafting $(\mathrm{CABG})$ is a favorable method of revascularization in patients with multivessel CAD (MVCAD). For patients with low SYNTAX score, percutaneous coronary intervention (PCI) is an acceptable revascularization strategy, although at the price of significantly higher rates of repeat revascularization [10]. Hybrid coronary revascularization (HCR) is a recently introduced, minimally invasive option for patients requiring revascularization for coronary lesions. Even though CABG is still the gold standard treatment for patients with MVCAD, HCR is also an accepted method of revascularization in this group of patients [11]. The POLMIDES study showed that $\mathrm{HCR}$ is feasible in selected patients with MVCAD referred for conventional CABG [12]. There are a few studies comparing QoL in patients with MV$\mathrm{CAD}$ treated with $\mathrm{HCR}$ and CABG. The aim of this study was to assess QoL in patients with MVCAD according to the mode of revascularization: $\mathrm{CABG}$ or HCR, using the generic SF-36 v.2 questionnaire.

\section{Methods}

From November 2009 to July 2012, 200 patients from the POLMIDES study with diagnosed MVCAD and referred for conventional CABG were randomized to $\mathrm{HCR}(\mathrm{n}=98)$ or CABG $(\mathrm{n}=102)$ in a 1:1 ratio. Before randomization the local heart team checked all the inclusion/exclusion criteria and the eligibility to perform $\mathrm{CABG}$ and $\mathrm{PCI}$ in all study participants, described previously in the POLMIDES study design [13]. Figure 1 shows the study flow chart. Six patients crossed over from HCR group to CABG group. During the follow-up observation 5 patients died. Fifteen patients from the HCR group and 21 patients from CABG group were lost to follow-up because of the incomplete HRQoL questionnaire or disagreement to undergo control angiography to assess the patency of grafts and restenosis in revascularized segments. Complete in-hospital and follow-up data were obtained from the target group: 159 (79.5\%) patients: 75 (76.5\%) patients in HCR group and in $84(82.4 \%)$ patients in CABG group. The primary endpoint was 
the change in HRQoL after the procedure. QoL was assessed by using the Polish version of the Medical Outcomes Study 36 questionaire version 2.0. A license for the use of SF-36 v.2 was obtained from OptumInsight Life Sciences, Inc., license number QM022262. The data from the HRQoL questionnaire were self-reported by the patients in the presence of the study psychologist. HRQoL was measured at two time points: hospital admission and at 12-months follow up. Subsequently the difference in HRQoL at this two time points was assessed. This questionnaire consists of 36 items or questions grouped into eight health-related aspects of the patient's life: Physical Functioning (PF), Role Physical (RF), Bodily Pain (BP), General Health (GH) Perceptions, Vitality (VT), Social Functioning (SF), Role-Emotional (RE) and Mental Health (MH). In addition, two summary scores are calculated using these eight scales: a physical component summary (PCS) consisting of $\mathrm{PF}+\mathrm{RF}+\mathrm{BT}+\mathrm{GH}$ and a mental component summary (MCS) consisting of $\mathrm{VT}+\mathrm{SF}+\mathrm{RE}+\mathrm{MH}$. Each of the subscales is scored on a scale of $0-100$, with higher scores indicating better outcomes. The PCS and MCS use normbased scoring and have a mean of 50 and a standard deviation of 10 . Also, there is one additional question comparing a patients' current health with that of the previous year. The demographic profile investigation questionnaire included questions on educational attainment, marital status, employment status and place of residence. Occurrence of major cardiac and cerebrovascular adverse events such as myocardial infarction, stroke, major bleeding and target vessel revascularization through the 12 -month period after randomization was also assessed. The definition of stroke, myocardial infarction, major bleeding, repeat revascularization and target vessel revascularization as described previously [13]. Diabetic patients were either treated with diet only, oral drugs or insulin injections. Dyslipidemia was defined as raised levels or cholesterol or triglycerides diagnosed before admission to hospital or during index hospitalization and was treated according to current guidelines. Obesity was diagnosed with body mass index over $30 \mathrm{~kg} / \mathrm{m}^{2}$.

The study conforms to the Declaration of Helsinki. The protocol was approved by the Ethics Committee of the Medical University of Silesia and all participants gave written, informed consent.

\section{Statistical analysis}

Continuous variables were presented as the mean \pm standard deviation or median and interquartile range.
Categorical variables were presented as frequencies and percentages. The normality assumption for continuous variables was checked by means of Shapiro-Wilk test. To test for differences between the CABG and HCR groups, the $\chi^{2}$ test and Fisher's exact test were used for categorical variables, and the Student t-test or Wilcoxon-MannWhitney test were used for continuous variables where appropriate.

Moreover, the difference in HRQoL between hospital admission and 12-months follow-up (for all domains) was categorized as an improvement in QoL when the difference was greater than 0 and a lack of improvement was observed otherwise. Next, such categorized variables were used in the multivariable logistic regression to evaluate the association between QoL improvement and the revascularization method adjusted for confounders. The stepwise selection method was used to build logistic regression models. A two-sided $\mathrm{p}$ value $<0.05$ was considered statistically significant. Statistical tests were performed with SAS version 9.4 software.

\section{Results}

Baseline characteristics are shown in Table 1. The vast majority in both groups were men of good global left ventricular systolic function (LVEF $50 \%$ ). Body mass index values in both groups were similar. About $30 \%$ of study subjects had diabetes, while more than $80 \%$ had hypertension. A mean SYNTAX score was 23.0 in the CABG group and 23.5 in the HCR group $(\mathrm{p}=0.76)$. Clinical events which occurred during hospital stay and follow-up are presented in Table 2. No patients died during in-hospital stay. There were no significant differences in in-hospital outcomes and during follow-up. The length of hospitalization was similar in both groups: 7.0 days (7.0-9.0) vs. 7.0 days (6.0-9.0), $\mathrm{p}=0.17$. For both treatment groups, assessment at 1 year revealed an improvement in QoL compared with the preintervention assessment in all 8 domains (Fig. 2). There was a substantial difference in HRQoL between treatment groups on admission. The general QoL of patients randomized to HCR was significantly higher at baseline and in 12 month follow-up than in patients randomized to CABG (59.2 vs. $51.3 ; \mathrm{p}<0.005)$ and (73.7 vs. 65.6 ; $\mathrm{p}<0.005)$. Both groups had the same QoL improvement: in HCR group: 13.5 (3.82-22.34) vs. CABG group: 10.48 (2.46-31.07); $\mathrm{p}=0.76$. Independent predictors of improvement in 6 out of 8 domains are presented in Figure 3. There were no independent 
Table 1. Baseline clinical characteristics.

\begin{tabular}{lccc}
\hline & CABG & HCR & P \\
\hline Age at randomization [years] & $63.5(59.0-71.0)$ & $64.0(57.0-69.0)$ & 0.43 \\
Male & $66(78.6 \%)$ & $58(77.3 \%)$ & 0.85 \\
Body mass index $\left[\mathrm{kg} / \mathrm{m}^{2}\right]$ & $28.9 \pm 4.02$ & $28.01 \pm 3.23$ & 0.13 \\
Hypertension & $68(81 \%)$ & $67(89.3 \%)$ & 0.14 \\
Diabetes & $25(29,8 \%)$ & $20(26.7 \%)$ & 0.67 \\
Dyslipiemia & $49(58.3 \%)$ & $41(54.7 \%)$ & 0.64 \\
Obesity & $37(44 \%)$ & $26(34.7 \%)$ & 0.23 \\
Current smoker & $32(38.1 \%)$ & $23(30.7 \%)$ & 0.33 \\
Atrial fibrillation & $5(6 \%)$ & $4(5.3 \%)$ & 0.87 \\
Ejection fraction $[\%]$ & $50(45-55)$ & $50(48-54)$ & 0.66 \\
Previous MI & $49(58.3 \%)$ & $37(49.3 \%)$ & 0.26 \\
Previous PCI & $35(41.7 \%)$ & $29(38.7 \%)$ & 0.7 \\
Previous stroke & $4(4.8 \%)$ & $3(4 \%)$ & 0.82 \\
Previous TIA & $2(2.4 \%)$ & 0 & 0.18 \\
Carotid artery disease & $10(11.9 \%)$ & $8(10.7 \%)$ & 0.81 \\
Stable angina & $69(82.1 \%)$ & $63(84 \%)$ & 0.76 \\
Unstable angina & $15(17.9 \%)$ & $12(16 \%)$ & $3(1-5)$ \\
Logistic EuroSCORE [points] & $3(2-5)$ & $23.5(18.0-26.5)$ & 0.76 \\
SYNTAX score & $23.0(19.3-26.0)$ & $40(53.7 \%)$ & 0.15 \\
2 vessel CAD & $45(53.6 \%)$ & 0.76 \\
3 vessel CAD & $39(46.4 \%)$ & 0.39
\end{tabular}

Categorical variables were expressed as frequencies (percentages), continuous variables were expressed as mean \pm standard deviation for normally distributed data and median ( $1^{\text {st }}$ and $3^{\text {rd }}$ quartile) for non-normally distributed data. CABG - coronary artery bypass grafting; $\mathrm{CAD}$ - coronary artery disease; HCR - hybrid coronary revascularization; LIMA — left internal mammary artery; MI - myocardial infarction; MIDCAB - minimally invasive coronary artery bypass; $\mathrm{PCl}$ - percutaneous coronary intervention; TIA — transient ischemic attack

Table 2. Clinical endpoints occurring in the hospital or after discharge.

\begin{tabular}{lccc}
\hline & CABG & HCR & P \\
\hline In-hospital outcomes & & & \\
Stroke & $0(0 \%)$ & $0(0 \%)$ & 1 \\
Perioperative MI & $4(4.8 \%)$ & $3(4 \%)$ & 0.87 \\
Major bleeding & $3(3.6 \%)$ & $1(1.3 \%)$ & 0.62 \\
Renal failure & $0(0 \%)$ & $0(0 \%)$ & 1 \\
Blood transfusion & $20(24 \%)$ & $11(15 \%)$ & 0.15 \\
Death & $0(0 \%)$ & $0(0 \%)$ & 1 \\
12 month follow up outcomes & & \\
Stroke & 0 & 0 & 1 \\
MI & 0 & $1(1.3 \%)$ & 0.47 \\
TVR & 0 & $2(2.7 \%)$ & 0.22 \\
Repeat hospitalization & $5(6 \%)$ & $6(8 \%)$ & 0.76 \\
\hline
\end{tabular}

CABG - coronary artery bypass grafting; HCR - hybrid coronary revascularization; $\mathrm{MI}$ - myocardial infarction; TVR — target vessel revascularization
Table 3. Health-related quality of life improvement rates in various populations.

\begin{tabular}{lccc}
\hline & CABG & HCR & P \\
\hline $\begin{array}{l}\text { Degree of education } \\
\text { Lower education }\end{array}$ & $83.7 \%$ & $90.6 \%$ & 0.38 \\
$\begin{array}{l}\text { Higher education } \\
\text { Marital status }\end{array}$ & $83.3 \%$ & $85.7 \%$ & 0.79 \\
$\begin{array}{l}\text { Relationship } \\
\text { Alone }\end{array}$ & $82 \%$ & $88.7 \%$ & 0.32 \\
Employment & $91 \%$ & $85 \%$ & 0.64 \\
Employed & & & \\
Unemployed & $83.3 \%$ & $80 \%$ & 0.82 \\
Place of living & $83.3 \%$ & $90.4 \%$ & 0.27 \\
City & & & \\
Rural & $83.1 \%$ & $86.7 \%$ & 0.61 \\
\hline
\end{tabular}

CABG - coronary artery bypass grafting; HCR — hybrid coronary revascularization 


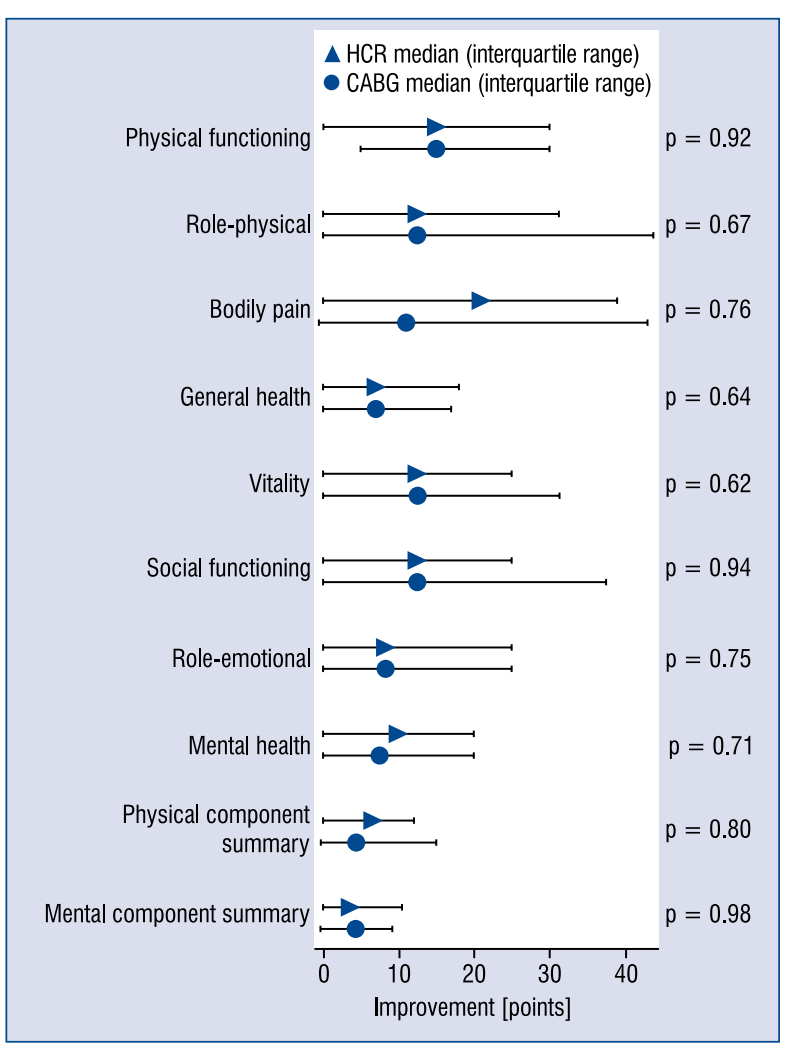

Figure 2. Improvement in health-related quality of life; CABG - coronary artery bypass grafting; HCR - hybrid coronary revascularization. predictors of improvement in domain RE, VT and PCS. Observation revealed that obesity worsened QoL in PF and MCS domain, female gender improved QoL in BP domain. In addition, analysis shows whether the degree of education (lower vs. higher), marital status (relationship vs. alone) employment (employed vs. unemployed) and place of living (city vs. village) had an influence on improvement in quality of life in both groups (HCR and $\mathrm{CABG}$ ). Depending on these factors, patients in both groups did not differ significantly (Table 3 ). Over $80 \%$ of all patients regardless of these factors felt improvement in HRQoL after the procedure.

\section{Discussion}

More and more frequently, physicians not only evaluate patient biological conditions, but also consider the impact of disease and treatment methods on patient functioning in everyday life. In recent years there has been an increasing use of instruments measuring QoL, particularly the HRQoL. The Medical Outcomes Study Short Form version 2 (SF-36 v.2) is considered to be a reliable, valid, concise generic measure of state of health that is widely used in heart disease [14]. According to Dempster and Donnelly SF-36 is the most

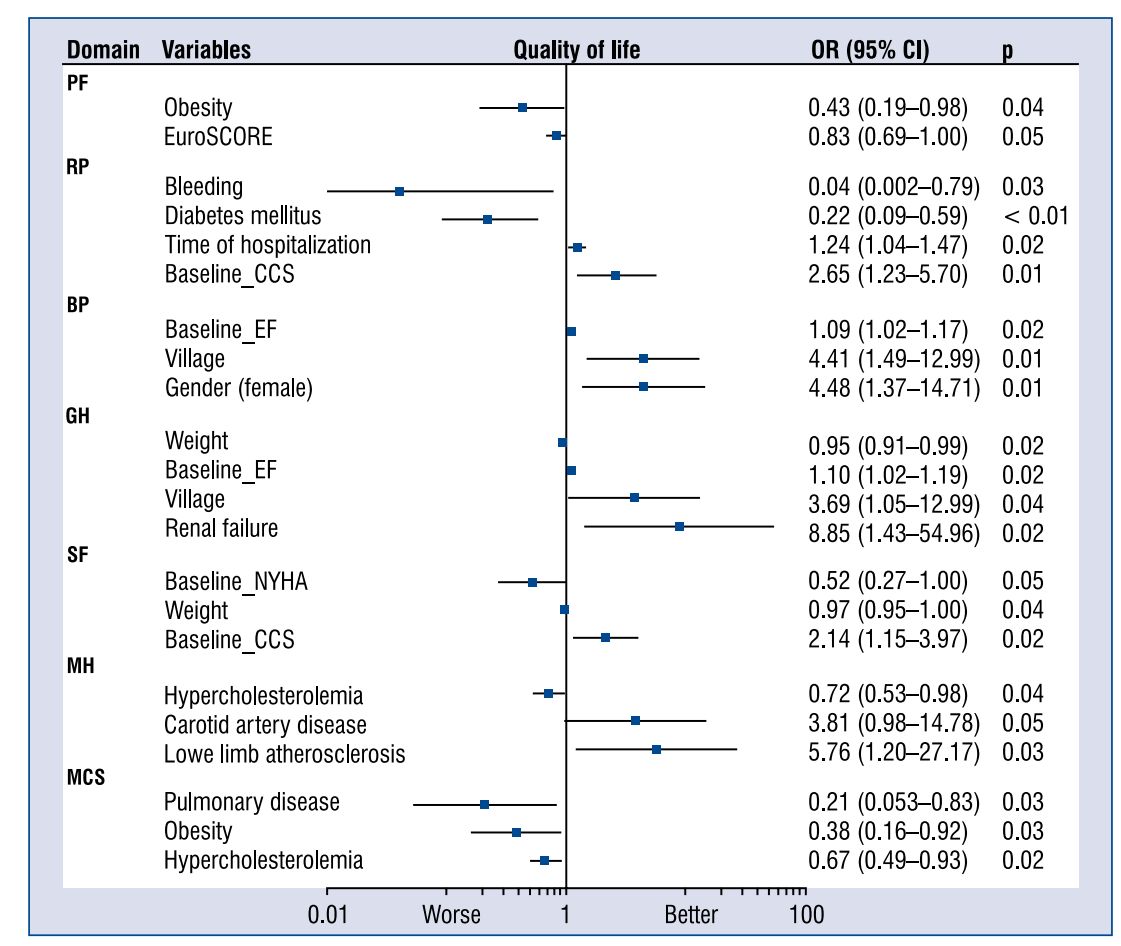

Figure 3. Independent predictors of improvement of quality of life — results of the logistic regression analysis; BP — bodily pain; CCS - Canadian Cardiovascular Society Class; $\mathrm{Cl}$ - confidence interval; EF — ejection fraction on admission; EF — ejection fraction; GH — General Health; MCS — Mental Component Summary; MH — Mental Health; NYHA - New York Heart Association; OR — odds ratio; PF — Physical Functioning; PVD — Peripheral Vascular Disease; RP — Role Physical; SF — Social Functioning; SF-36 — Short Form 36 Health Survey. 
appropriate generic instrument to assess HRQoL in patient with ischemic heart disease [15]. They compared the validity, reliability, and sensitivity of the SF-36 with other generic questionnaires such as the Nottingham Health Profile and the Sickness Impact Profile for patients with CAD. Numerous studies including RITA and CABRI trial have examined HRQoL after PCI or CABG and demonstrated significant improvements in HRQoL for patients undergoing percutaneous or surgical revascularization which compare baseline and also compare with medical therapy $[16,17]$. This became the basis for research of QoL. Angelini et al. [18] reported the first HCR procedure in 1996. The concept was based on performing a minimally invasive thoracotomy LIMA-LAD anastomosis and balloon angioplasty of non-LAD vessels. Hybrid coronary revascularization is a promising technique that combines advantages of both: CABG and PCI. More and more studies confirm safety and effectiveness of HCR method for complete revascularization in selected patients with MVCAD. There are few studies analyzing the QoL in this group of patients. This study is one of the largest prospective reports including $\mathrm{HRQOL}$ in patients undergoing HCR in comparison to CABG. The present study showed that patients participating in the POLMIDES study in a 12-month follow up achieved a significant improvement in QoL, regardless of method of treatment. Improvement was measured as an increase in the QoL, and did not significantly differ for both groups in each of 8 domains. The level of education, marital status, employment and place of living did not influence the improvement of QoL in either group. When comparing the QoL scores, both revascularization strategies were equally effective. According to Bachinsky et al. [19], who compared same-setting robotic-assisted hybrid coronary revascularization to off-pump coronary artery bypass, QoL at 30 days measured using SF-12 questionnaire was better in the hybrid group (physical QoL score $32.8 \pm 10.4$ vs. $41.6 \pm 10.3, \mathrm{p}=0.009)$. De Cannie're et al. [20] performed a retrospective comparison of $20 \mathrm{pa}$ tients with double-vessel CAD treated with staged hybrid revascularization with a matched-group of patients who underwent on-pump CABG. Quality of life was significantly better in the MIDCAB group at 6 weeks, whereas the 2 -year subsequent questionnaire showed no significant difference in QoL between the groups. This short-term followup can be a result of an open chest technique or adverse reactions often caused by extracorporeal circulation during CABG. Previous studies suggest that patients undergoing MIDCAB procedures experience no advantage with regard to postoperative pain compared to conventional CABG. A similar observation was made in POLMIDES study at 1 year and observed no significant differences in HRQoL between the two modes of revascularization. Finally, although CABG remains the gold standard, HCR offers the same effective method of revascularization, with similar QoL. However, their use is restricted to a very limited group of patients. As the population of the patients with MVCAD referred for $\mathrm{CABG}$ are getting older with greater comorbidities, a less invasive technique should be explored. No less important is QoL after these alternative procedures. Despite an initially more demanding procedure, CABG patients achieved equal level of HRQoL when compared with HCR patients.

\section{Limitations of the study}

This study had several limitations. First, the influence of coexisting depressive disorders on QoL, as QoL was not taken into consideration and was also influenced by perioperative anxiety and depression. Despite these factors, the study population is currently the largest published cohort of patients comparing these two methods of revascularization and it also reported on QoL. Second, some patients completed the questionnaire after knowing their randomization group. It seems possible that the emotional aspect may play a role in perioperative period.

\section{Conclusions}

Patients with MVCAD after both modes of revascularization (CABG and $\mathrm{HCR}$ ) showed significant improvements in QoL from baseline to 1-year follow-up in all domains. According to the literature SF-36 v.2 is one of the most appropriate generic instruments to assess HRQoL in patients with ischemic heart disease.

\section{Acknowledgements}

Data presented in this manuscript are based on POLMIDES (HYBRID) study, which was funded by the Ministry of Science and Higher Education of Poland, operated by the National Research and Development Center, Project No. R 13008406.

Conflict of interest: None declared 


\section{References}

1. Gardner AW, Montgomery PS, Ritti-Dias RM, et al. Exercise performance, physical activity, and health-related quality of life in participants with stable angina. Angiology. 2011; 62(6): 461-466, doi: 10.1177/0003319711399897, indexed in Pubmed: 21421630.

2. Greenfield S, Nelson EC. Recent developments and future issues in the use of health status assessment measures in clinical settings. Med Care. 1992; 30(5 Suppl): MS23-MS41, indexed in Pubmed: 1583935.

3. Wilson IB, Cleary PD. Linking clinical variables with healthrelated quality of life. JAMA. 1995; 273(1): 59-65, indexed in Pubmed: 7996652.

4. Lam CLK. What is health-related quality of life (HRQOL)? Hong Kong Practitioner. 1997; 19: 505-506.

5. Ware JE, Snow KK, Kosinski M. et al., SF-36 Health Survey: Manual and interpretation guide. The Health Institute, New England Medical Centre, Boston 1993.

6. Ware JE, Kosinski MA, Keller SD. SF-36 Physical and Mental Component Summary Measures: a User's Manual. The Health Institute New England Medical Center, Boston 1994.

7. Cohen DJ, Van Hout B, Serruys PW, et al. Quality of life after $\mathrm{PCI}$ with drug-eluting stents or coronary-artery bypass surgery. N Engl J Med. 2011; 364(11): 1016-1026, doi: 10.1056/NEJMoa1001508, indexed in Pubmed: 21410370.

8. Mathai SC, Suber T, Khair RM, et al. Health-related Quality of Life and Survival in Pulmonary Arterial Hypertension. Ann Am Thorac Soc. 2016; 13(1): 31-39, doi: 10.1513/AnnalsATS.2014125720C, indexed in Pubmed: 26492065.

9. Sportelli E, Regesta T, Salsano A, et al. Does patient-prosthesis mismatch after aortic valve replacement affect survival and quality of life in elderly patients? J Cardiovasc Med (Hagerstown). 2016; 17(2): 137-143, doi: 10.2459/JCM.0000000000000292, indexed in Pubmed: 26237424.

10. Windecker S, Kolh P, Alfonso F, et al. 2014 ESC/EACTS Guidelines on myocardial revascularization. The Task Force on Myocardial Revascularization of the European Society of Cardiology (ESC) and the European Association for Cardio-Thoracic Surgery (EACTS). Eur Heart J. 2014; 35: 2821-2830.

11. Gosev I, Leacche M. Hybrid coronary revascularization: the future of coronary artery bypass surgery or an unfulfilled promise? Circulation. 2014; 130(11): 869-871, doi: 10.1161/ /CIRCULATIONAHA.114.011857, indexed in Pubmed: 25055813.

12. Gąsior M, Zembala MO, Tajstra M, et al. Hybrid revascularization for multivessel coronary artery disease. JACC Cardiovasc Interv. 2014; 7(11): 1277-1283, doi: 10.1016/j.jcin.2014.05.025, indexed in Pubmed: 25459040.

13. Zembala M, Tajstra M, Zembala M, et al. Prospective randomised pilOt study evaLuating the safety and efficacy of hybrid revascularisation in MultI-vessel coronary artery DisEaSe (POLMIDES): study design. Kardiol Pol. 2011; 69(5): 460-466, indexed in Pubmed: 21594832.

14. Failde I, Ramos I. Validity and reliability of the SF-36 Health Survey Questionnaire in patients with coronary artery disease. J Clin Epidemiol. 2000; 53(4): 359-365, doi: 10.1016/s08954356(99)00175-4.

15. Dempster M, Donnelly M. Measuring the health related quality of life of people with ischaemic heart disease. Heart. 2000; 83(6): 641-644, indexed in Pubmed: 10814620.

16. Pocock SJ, Henderson RA, Seed P, et al. Quality of life, employment status and anginal symptoms after coronary angioplasty or bypass surgery. Circulation. 1996; 94: 135-142.

17. Pocock SJ, Henderson RA, Clayton T, et al. Quality of life after coronary angioplasty or continued medical treatment for angina: three-year follow-up in the RITA-2 trial. J Am Coll Cardiol. 2000; 35: 907-914.

18. Angelini GD, Wilde P, Salerno TA, et al. Integrated left small thoracotomy and angioplasty for multivessel coronary artery revascularisation. Lancet. 1996; 347(9003): 757-758, indexed in Pubmed: 8602013.

19. Bachinsky WB, Abdelsalam M, Boga G, et al. Comparative study of same sitting hybrid coronary artery revascularization versus offpump coronary artery bypass in multivessel coronary artery disease. J Interv Cardiol. 2012; 25(5): 460-468, doi: 10.1111/j.15408183.2012.00752.x, indexed in Pubmed: 22758203.

20. de Cannière D, Jansens JL, Goldschmidt-Clermont P, et al. Combination of minimally invasive coronary bypass and percutaneous transluminal coronary angioplasty in the treatment of doublevessel coronary disease: Two-year follow-up of a new hybrid procedure compared with "on-pump” double bypass grafting. Am Heart J. 2001; 142(4): 563-570, doi: 10.1067/mhj.2001.118466, indexed in Pubmed: 11579343. 